Mein Jahrhundertbuch

Herausgegeben von Iris Radisch

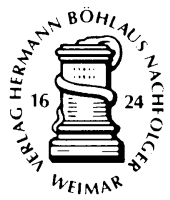




\section{Mein Jahrhundertbuch}

51 namhafte Autoren stellen ihr

Lieblingswerk des 20. Jahrhunderts vor

Ein ZEIT-Buch

Herausgegeben von Iris Radisch

2000

Verlag Hermann Böhlaus Nachfolger Weimar 
Die Deutsche Bibliothek - CIP-Einheitsaufnahme

Mein Jahrhundertbuch :

51 namhafte Autoren stellen ihr Lieblingswerk

des 20. Jahrhunderts vor ; ein ZEIT-Buch /

hrsg. von Iris Radisch.

- Weimar : Verlag Hermann Böhlaus Nachfolger, 2000

ISBN 978-3-7400-1141-3

ISBN 978-3-7400-1141-3

ISBN 978-3-476-02728-3 (eBook)

DOI 10.1007/978-3-476-02728-3

Dieses Werk einschließlich aller seiner Teile ist urheberrechtlich geschützt. Jede Verwertung außerhalb der engen Grenzen des Urheberrechtsgesetzes ist ohne Zustimmung des Verlages unzulässig und strafbar. Das gilt insbesondere für Vervielfältigungen, Übersetzungen, Mikroverfilmungen und die Einspeicherung und Verarbeitung in elektronischen Systemen.

(c) 2000 Springer-Verlag GmbH Deutschland

Ursprünglich erschienen bei Verlag Hermann Böhlaus Nachfolger Weimar 2000 


\section{Inhalt}

Vorwort von Iris Radisch . . . . . . . . . . . . IX

Franz Kafka, Der Prozess (Louis Begley) . . . . . . . . 1

James Joyce, Ulysses (Salman Rushdie) . . . . . . . . . . 5

Christoph David Friedrich Weinland, Rulaman

(Günter Herburger) . . . . . . . . . . . . . . . . . 8

Robert Musil, Der Mann ohne Eigenschaften

(Milan Kundera) . . . . . . . . . . . . . . . . . 11

Max Horkheimer / Theodor W. Adorno, Dialektik der

Aufklärung (Peter Bürger) . . . . . . . . . . . . . . . 14

Thomas Mann, Buddenbrooks (Siegfried Lenz) . . . . . 17

Samuel Beckett, Der Namenlose (Wilhelm Genazino) . . . 20

Tobias Wolff, In der Armee des Pharaos (John le Carré) . . 23

Albert Camus, Der Fremde (Péter Nádas) . . . . . . . . . 24

Marcel Proust, Auf der Suche nach der verlorenen Zeit

(Cees Nooteboom) . . . . . . . . . . . . . . 28

Ezra Pound, Pisaner Cantos (Raoul Schrott) . . . . . . . 33

Christoph Wilhelm Aigner, Mensch. Verwandlungen

(Sarah Kirsch) . . . . . . . . . . . . . . . . . . 37

Erich Mater, Rückläufiges Wörterbuch der deutschen

Gegenwartssprache (Oskar Pastior) . . . . . . . . . . . 40

Alexander Solschenizyn, Der Archipel Gulag

(Wolfgang Hilbig) . . . . . . . . . . . . . . . . . 43

Diverse Bücher (Valeria Narbikova) . . . . . . . . . . . 45

Gerard Manley Hopkins, Journal (Friederike Mayröcker) . 47

Über das Nichtlineare und Georges Pérec (Milorad Pavic) 49

Ernst Herbeck, Im Herbst da reiht der Feenwind

(Leo Navratil) . . . . . . . . . . . . . . . . . . . . 52 
Über Ettore Schmitz, der sich Italo Svevo nannte (Claudio Magris) . . . . . . . . . . . . . . . . . 55

Albert Vigoleis Thelen, Die Insel des zweiten Gesichts (Maarten 't Hart) . . . . . . . . . . . . . . . . 61

Sigmund Freud, Vorlesungen zur Einführung in die Psychoanalyse (Richard Rorty) . . . . . . . . . . . . . . . 63 Friedrich Dürrenmatt, Stoffe (Dieter Bachmann) . . . . . 67 Anatole France, Auf dem weißen Felsen (Jürgen Engler) . . 70 Martin Heidegger, Sein und Zeit (Michael Theunissen) . . 73 Winsor Mc Cay, Little Nemo (Brigitte Kronauer) . . . . . 76 Allen Ginsberg, Howl und Kaddish (István Eörsi) . . . . . 79 Franz Kafka, Das Schloss (Georges-Arthur Goldschmidt) . 83 Friedrich A. von Hayek, Der Weg zur Knechtschaft (John R. Searle) . . . . . . . . . . . . . . . . . . . . 86 Bertolt Brecht, Buckower Elegien (Hans Jürgen Syberberg) 90 Andrej Tarkowskij, Die versiegelte Zeit (Gerhard Roth) . . 94 Michail A. Bulgakow, Der Meister und Margarita (Dževad Karahasan) . . . . . . . . . . . . . . . . . . . 98 Jaroslav Hašek, Die Abenteuer des braven Soldaten Schwejk (Daniil Granin) . . . . . . . . . . . . . . . . . . . 103 Georg K. Glaser, Geheimnis und Gewalt (Peter Härtling) . 107 Louis-Ferdinand Céline, Reise ans Ende der Nacht (László F. Földényi) . . . . . . . . . . . . . . . 111 Juan Rulfo, Pedro Páramo (Urs Widmer) . . . . . . . . . 115 Anthony Burgess, Der Fürst der Phantome (Nigel Barley) . 118 Franz Kafka, Das Schloss (László Krasznahorkai) . . . 122 Albert Camus, Die Pest (Lars Gustafsson) . . . . . . . . 126 Johannes Bobrowski, Levins Mühle (Ingo Schulze) . . . . 132 Jean-Paul Sartre, Der Ekel (Stewart O'Nan) . . . . . . . 138 Christian Morgenstern, Galgenlieder (Walter Kempowski) 141 Antonio Gramsci, Die Südfrage (Fabrizia Ramondino) . . 145 Franz Kafka, Tagebücher 1910-1923 (George Tabori) . . . 151 Alexander Solschenizyn, Der Archipel Gulag (Ivan Klíma) 152 
Vladimir Nabokov, Lolita (Viktor Jerofejew) . . . . . . . 154 Primo Levi, Ist das ein Mensch? (Günter Kunert) . . . . . 158 Über Marcel Proust (Nadine Gordimer) . . . . . . . . . . 161 Ernst Barlach, Der gestohlene Mond (Adolf Muschg) . . . 162 Henri Alain-Fournier, Der große Meaulnes (Ludwig Harig) 166 Robert Musil, Der Mann ohne Eigenschaften (Katarina Frostenson) . . . . . . . . . . . . . 169 Witold Gombrowicz, Pornographie (Péter Esterházy) . . . 173

Die Rezensenten . . . . . . . . . . . . . . . . 177 Inhalt (alphabetisch) $\ldots \ldots \ldots 181$ 


\section{Vorwort}

Die Frage war so größenwahnsinnig wie unbeantwortbar: Nennen Sie mir, auf die Gefahr, ungerecht zu sein, ein Buch, das für Sie das wichtigste Buch des 20. Jahrhunderts ist? Dennoch haben wir sie Schriftstellern und Gelehrten auf der ganzen Welt ins Haus geschickt. Ein Jahr lang, bis zur letzten Ausgabe des nun vergangenen Jahrtausends, haben wir in der ZEIT Bilanz gezogen. Welche Bücher werden bleiben? Welche Bücher werden über die Schwelle der Jahrtausendwende getragen, wie sieht unsere eiserne Bücherration aus? Auch wenn jeder weiß, dass Bilanzen, die man nicht selbst gefälscht hat, nie stimmen, war die Frage nach einer Bibliothek des 20. Jahrhunderts zu verlockend.

Die Gegenfragen lagen auf der Hand und bei uns schnell im Briefkasten. Warum durfte man nur ein einziges Jahrhundertbuch nennen? Wer kann das schon? Literatur, meldete Peter Rühmkorf von der nahen Elbe ins Pressehaus am Speersort, sei schließlich ein »riesiger Resonanzraum«, wie sich da auf ein einziges »Urknallbuch « beschränken? Und warum nur ein Buch aus dem 20. Jahrhundert? Warum nicht eines beispielsweise von Kleist, schrieb Dževad Karahasan aus Sarajevo, denn war Kleist nicht in tiefster Seele bereits ein Autor des 20. Jahrhunderts? Und überhaupt: "Doesn't it seem quite fabricated such a choosing«, raunzte Richard Ford aus New Orleans. Und Christoph Ransmayr schickte, animiert von der unbeantwortbaren Frage, aus Irland wunderbare Erzählungen von Teigbuchstaben, die aus einer fettglänzenden, längst verdampften Suppe aufsteigen, aber keinen Buchtitel ergeben, berichtete von den russischen und griechischen Büchern seines Vaters, vom Radio seines Onkels, auf dem zwei Steinfiguren standen und sich gegen einen 
kleinen Bücherstapel stemmten und gab uns auf wunderbare Weise zu verstehen, dass die Erzählung von seinem Jahrhundertbuch eine sehr persönliche Sache, keine Angelegenheit von zwei Schreibmaschinenseiten sein kann.

Und dennoch zielt die Frage nach dem persönlichen Jahrhundertbuch genau da hinein: ins Herz jedes einzelnen Lesers. Denn welche andere Legitimation sollte ein Kanon der Weltliteratur des 20. Jahrhunderts haben, als die eines liebenden lesenden Herzens? Eine imaginäre Zentralbibliothek, zusammengestellt von berühmten Dichtern und Denkern, ist natürlich kein verbindlicher Kanon. Keine Jury hat darüber gewacht, dass die anerkannten großen Meisterwerke des Jahrhunderts in dieser Bibliothek enthalten sind. Niemand hat für den Proporz, für Ausgleich und Repräsentanz gesorgt. Darin unterscheidet sich die Jahrhundertbuch-Bibliothek von der ZEIT-Bibliothek der 100 Bücher, die vor genau zwanzig Jahren Furore machte. Damals haben sich fünf weise Herren die Köpfe darüber zerbrochen, welche 100 Bücher in einen Kanon der Weltliteratur hineingehören. Ein Zwei-Jahres-Plan wurde erstellt und Woche für Woche ausgeführt, eine literaturkritische Planwirtschaft wurde betrieben, die den Vorzug der relativen Gerechtigkeit hatte.

Die Bibliothek der Jahrhundertbücher ist dagegen ungerecht. Sie folgt keinem Plan, außer dem der Neigungen und Vorlieben. War die ZEIT-Bibliothek der 100 Bücher ein sorgsam angelegter Park, so ist dies ein wildwuchernder Garten der Leselüste. Der Weg, den der Leser durch diesen Garten zurücklegt, beginnt - und das ist sicher kein Zufall - bei Franz Kafka, biegt zielgerade ab zu Joyce, führt zu Robert Musil, landet weich bei Thomas Mann, macht zweimal Rast bei Albert Camus, taucht ein in den Strom der verlorenen Zeit des Marcel Proust und kehrt immer wieder zu Kafka zurück. Als habe eine unsichtbare Hand im Geheimen doch Regie geführt, zeichneten sich auf der Landkarte des Lesens deutliche Knotenpunkte und 
vor allem Grenzen ab: Obwohl Autoren aus aller Welt nach ihrem Lieblingsbuch befragt wurden, ist die Heimat der Jahrhundertbücher vor allem Zentraleuropa, insbesondere Deutschland, Frankreich, Russland und Italien. Gleichwohl herrscht in diesem Kernland der Literatur die Anarchie des Herzens. Kindheitsbücher, Wörterbücher, Bücher von Freunden und Vorbildern, Bücher, die das Leben in wichtigen Jahren begleitet haben, Bücher von großer regionaler Bedeutung stehen neben den anerkannten Wackersteinen der Weltliteratur. Was ein Jahrhundertbuch ausmacht, entscheiden nicht Experten, sondern die Zufälle und Bedingungen des privaten Lebens.

Auch dies kann man nebenbei erfahren: Den großen Dichtern und Denkern geht es nicht anders als jedem Leser: Er liest, was er liebt, und er liebt vor allem, was ihm, nein kein Kanon, keine Empfehlungsliste, sondern das Leben selber nahe gebracht hat. Manchmal ist es wie bei Péter Esterházy eine alte Tante, die sich abfällig über Gombrowicz äußert - Wissen Sie Peterrr, er ist ein sehr großer Schriftsteller und sehr unangenehm - und die Neugier entfacht. Manchmal ist es ein Band von Louis-Ferdinand Céline, der wie eine Briefbombe in das Leben des ungarischen Philosophen László F. Földényi fällt. Der brave Schwejk hat Daniil Granin schon in manch schwerer Lebensminute ein Bier angeboten. Der Fremde von Albert Camus liegt Peter Nadás schon deswegen am Herzen, weil er im Jahr seines Erscheinens geboren ist. Und auf Little Nemo ist Brigitte Kronauer beim Stöbern in einer Hamburger Buchhandlung gestoBen. Häufig sind die Lieblingsbücher die, die man seit der Jugend mit sich herumträgt. So hat Michael Theunissen Sein und Zeit von Martin Heidegger schon als 18-Jähriger in einem Zug verschlungen. Und Günter Herburger zieht noch immer jenes Werk allen anderen vor, das er als Kind in der eiskalten Kammer des Nachts mit der Taschenlampe unter der Bettdecke gelesen hat. 
Erstaunlich auch, dass im Zweifel das wichtigste, das letzte Buch, das man aus dem vergangenen Jahrhundert mitnehmen möchte, doch ein Roman ist. Gewählt werden durfte alles: Gedicht, Essay, Erzählung, Drama, Aphorismus und philosophisches Werk. Und doch stehen am Ende des Jahrhunderts der großen Ideologien kaum theoretische Werke in der Endauswahl unserer Autoren. Freuds Vorlesungen zur Einführung in die Psychoanalyse, Die Dialektik der Aufklärung von Max Horkheimer und Theodor W. Adorno, Antonio Gramscis Südfrage, Heideggers Sein und Zeit und Der Weg zur Knechtschaft von Friedrich A. Hayek sind alles, was vom Jahrhundert der Hannah Ahrendt und Rosa Luxemburg, des George Lukács und Norbert Elias, des Edmond Husserl und des Ludwig Wittgenstein, des Max Weber und des Niklas Luhmann übrig geblieben sind. Woraus man mit aller Vorsicht schließen könnte, dass das Haltbarkeitsdatum theoretischer Texte ein besonders endliches ist und literarische Weltdeutungen den abstrakten auf lange Sicht überlegen sind.

"Das wichtigste Buch des Jahrhunderts«, scheibt Richard Rorty in seinem Beitrag über Freud, "müsste das Werk sein, dem zukünftige Historiker einhellig die größten Veränderungen im Selbstbild der menschlichen Rasse zuschreiben werden«. Wenn dies stimmt, so ist das Selbstbild des Menschen im Roman noch immer der stärkste Indikator für die Umwälzungen und Revolutionen des Menschenbildes.

Doch was für ein Menschenbild ist es, das die Jahrhundertbibliothek zeichnet? Auffallend ist, dass der Mensch in der Bibliothek des 20. Jahrhunderts schon recht betagt ist. Die jüngste und allerjüngste Literatur kommt nicht vor, die deutschsprachige Nachkriegsliteratur fehlt, abgesehen von Johannes Bobrowski und dem späten Dürrenmatt, beinahe vollständig. Kein Uwe Johnson, kein Peter Weiss, keine Ingeborg Bachmann, aber auch kein Günter Grass, keine Christa Wolf, kein Max 
Frisch, von den noch Jüngeren ganz zu schweigen. Die moderne Literatur wird einzig von Allen Ginsberg und Georges Perec vertreten. Die junge osteuropäische Literatur endet bei Nabokov und Gombrowicz. Insofern ist diese kleine Bibliothek eher konservativ zu nennen. Obwohl sie dunkle, abgründige Autoren wie Louis-Ferdinand Céline, Ezra Pound oder (eine besondere Überraschung) Andrej Tarkowskij nicht ausschließt, überwiegen doch die großen Realisten des bürgerlichen Zeitalters: Marcel Proust, Thomas Mann und Robert Musil auf der Seite der großen ironischen Chronisten; Franz Kafka, Samuel Beckett, Bertolt Brecht, Albert Camus, Jean-Paul Sartre auf der Seite der Minimalisten und Moralisten.

»Den Roman des 20. Jahrhunderts haben Joyce, Proust und Kafka erfunden «, schreibt Salman Rushdie und hat damit im Grunde den geheimen Horizont dieser erlesenen Schriftstellerbibliothek umrissen: er ist europäisch, realistisch, bürgerlich und - pardon - männlich. Insofern hat sich im Nachhinein betrachtet unter der Hand doch etwas wie ein Kanon herauskristallisiert, der gegen alle Katastrophen der Historie resistent war. Noch immer holt man sich Auskunft über die Schrecken des Jahrhunderts am liebsten bei Kafka, Camus und Beckett. Noch immer ist das gesellschaftliche Universum des 20. Jahrhunderts das seiner melancholischen Apologeten, sprich das von Mann, Musil, Proust oder Svevo.

Und doch will auch in diesen Kanon der hochentwickelten bürgerlichen Katastrophenliteratur zumindest ein Titel, der in diese Bibliothek gleich zwei Mal aufgenommen wurde, nicht ganz passen. Es ist der Archipel Gulag von Alexander Solschenizyn, den Ivan Klíma als den schlagkräftigsten Beweis dafür anführt, dass Literatur Reiche erschüttern kann, die völlig unerschütterlich scheinen. Ohne dieses Buch, schreibt auch Wolfgang Hilbig, hätten sich die kommunistischen Zwangssysteme des 20. Jahrhunderts vermutlich nicht so schnell und so 
umfassend aufgelöst. "Wem das Ende der kommunistischen Illusion noch immer unbegreiflich oder überraschend erscheint, der lässt dieses Buch außer Acht«, meint Hilbig. Dieses Buch begleitet die Geschichte nicht, es macht Geschichte, es ist "zutiefst menschlich in seinem Bild der Unmenschlichkeit", schreibt Ivan Klíma, "und so charakteristisch für das 20. Jahrhundert, dass es vielleicht das Buch des Jahrhunderts ist."

Wenn es also einen gemeinsamen Nenner jenseits der puren Vorlieben und biographischen Zufälligkeiten in dieser Bibliothek gibt, so ist es dieser Glaube, dass Literatur Reiche erschüttern kann, innere und äußere. Es ist der Glaube an die Literatur als einer Kraft, die uns verändert, die uns etwas lehrt, in der wir uns wieder finden, die Gemeinsamkeit stiftet. Die Bibliothek des 21. Jahrhunderts mag ganz anderen Gesetzen gehorchen. Unsere erzählt von den großen Träumen des vergangenen Jahrhunderts, von der Pracht und Herrlichkeit einer Literatur, die mehr sein wollte als ansprechender Zeitvertreib. Dieser Ernst macht die kleine Bibliothek, auch in ihren Seitenarmen und skurrilen Außenposten, zu einem Dokument. Ein Beweis dafür, dass es einmal eine größenwahnsinnge Zeit gegeben hat, in der das Lesen noch geholfen hat. 